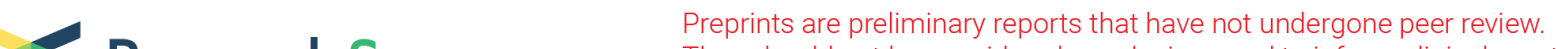 Research Square
or referenced by the media as validated information.
}

\section{Trehangelins ameliorate inflammation-induced skin senescence by suppressing the epidermal YAP- CCN1 axis}

Mami Yokota ( $\square$ mami1704@fancl.co.jp)

FANCL Research Institute, FANCL Corporation

\section{Yoshiyuki Kamiya}

FANCL Research Institute, FANCL Corporation

\section{Tamie Suzuki}

FANCL Research Institute, FANCL Corporation

\section{Shinsuke Ishikawa}

Department of Plastic and Aesthetic Surgery, Kitasato University School of Medicine

\section{Akira Takeda}

Department of Plastic and Aesthetic Surgery, Kitasato University School of Medicine

\section{Shinya Kondo}

FANCL Research Institute, FANCL Corporation

\section{Takeshi Tohgasaki}

FANCL Research Institute, FANCL Corporation

\section{Takuji Nakashima}

Research Organization for Nano \& Life Innovation, Waseda University

\section{Yoko Takahashi}

Ōmura Satoshi Memorial Institute, Kitasato University

\section{Satoshi Ōmura}

Ōmura Satoshi Memorial Institute, Kitasato University

\section{Tetsuhito Sakurai}

FANCL Research Institute, FANCL Corporation

\section{Research Article}

Keywords: Trehangelins, Sensitive skin, Cellular Communication Network factor 1 (CCN1), senescence, epidermal differentiation, extracellular matrix

Posted Date: July 30th, 2021

DOI: https://doi.org/10.21203/rs.3.rs-737551/v1 
License: (c) (i) This work is licensed under a Creative Commons Attribution 4.0 International License. Read Full License 


\section{Abstract}

Trehangelins (THG) are newly identified trehalose compounds derived from broth cultures of an endophytic actinomycete, Polymorphospora rubra. THG are known to suppress Cellular Communication Network factor 1 (CCN1), which regulates collagen homeostasis in the dermis. Although the physical properties of THG suggest a high penetration of the stratum corneum, the effect of THG on the epidermis has not been reported. Here we describe a possible mechanism involved in skin aging focusing on the effect of THG on epidermal CCN1. This study shows that: 1) THG suppress epidermal CCN1 expression by inhibiting the translocation of Yes-Associated Protein (YAP) to nuclei. 2) Epidermal CCN1, localized at the basement membrane, regulates the balance between the growth and differentiation of keratinocytes. 3) Keratinocytes secrete more CCN1 than fibroblasts, which leads to disruption of the basement membrane and extracellular matrix components. 4) The secretion of CCN1 from keratinocytes is increased by ultraviolet B exposure, especially in aged keratinocytes, and deteriorates the elastic fiber structures in the underlying dermis. 5) Topical application of THG ameliorates the structure of the basement membrane in ex vivo human skin explants. Taken together, THG might be a promising treatment for aged skin by suppressing the aberrant YAP-CCN1 axis.

\section{Introduction}

Trehangelins (THG) are novel natural products derived from broth cultures of an endophytic actinomycete strain, Polymorphospora rubra K07-0510 [1]. THG consist of a trehalose moiety and two angelic acid moieties, and show a potent inhibitory activity against the hemolysis of red blood cells induced by lightactivated pheophorbide A [1]. We previously reported that THG suppresses Cellular Communication Network factor 1 (CCN1), a ubiquitously expressed matricellular protein, in dermal fibroblasts [2].

CCN1 is a member of the CCN family, deficiencies of which cause a failure in vasculogenesis during embryonic development [3]. Four structurally distinct domains characterize the function of CCN1: an insulin-like growth factor binding protein domain, a von Willebrand type $\mathrm{C}$ repeat domain, a thrombospondin type 1 repeat domain and a C-terminal cysteine-knot motif domain [4]. CCN1 promotes cell proliferation, survival and angiogenesis by binding to av $\beta 3$-integrin, and it induces apoptosis and senescence through a6 31 -integrin and proteoglycans [4]. In the dermis, levels of CCN1 increase with intrinsic aging and photoaging, and then drive senescence via the dysregulation of collagen metabolism [5-7], which forms the age-associated dermal microenvironment [8]. In contrast, the level of CCN1 is elevated in the entire epidermis of the lesional skin of psoriasis patients and promotes the production of Interleukin-8 (IL-8), IL-1 $\beta$ and C-C Motif Chemokine Ligand 20, which drives psoriatic inflammation [9-11].

Sensitive skin is another skin condition involved in epidermal inflammation. Generally, sensitive skin is thought to be hypersensitive to environmental factors such as ultraviolet (UV) radiation, dryness, pollution, etc. [12] and is vulnerable to skin problems. An epidemiological study showed that subjects complaining about "sensitive" or "very sensitive" skin were 2-4 times more likely to declare suffering from dermatosis [13]. Since the concept of "sensitive skin" emerged in the 1970's [14], the population has 
steadily increased and has become an aging-conscious generation. Although often transient and in many cases unaccompanied by visible dermatological responses, sensitive skin can significantly affect the quality of life [15]. Thus, understanding the behavior of factors involved in the pathogenesis of skin is important.

Although the physical properties of THG suggest a high penetration of the stratum corneum, the effect of THG on epidermal CCN1 in normal non-psoriatic skin remains unknown. The fact that CCN1 is associated both with epidermal inflammatory skin diseases and with dermal collagen homeostasis led us to hypothesize that CCN1 might have an important role in the inflammation and aging of normal human skin. Thus, the aim of this study was to clarify the characteristic roles of CCN1 in the epidermis and to identify an effective skin care solution that could be used to treat sensitive and aged skin.

The results of this study demonstrate that THG suppresses epidermal CCN1 by retaining Yes-Associated Protein (YAP) in the cytosol, which prevents its translocation to nuclei. Further, we demonstrate that excess secreted CCN1 in aged and stress-stimulated keratinocytes may induce an imbalance in epidermal growth and differentiation, in inflammation and on the disruption of the extracellular matrix. We propose a possible mechanism for sensitive skin aging driven by CCN1 and a skin care solution by the topical application of THG.

\section{Results}

\section{THG suppresses the expression of CCN1 in keratinocytes by inhibiting the translocation of YAP to the} nucleus. THG are newly identified trehalose compounds derived from broth cultures of an endophytic actinomycete strain, Polymorphospora rubra K07-0510 [1]. THG consists of a trehalose moiety and two angelic acid moieties (Fig. 1a). We previously reported that THG decreases CCN1 expression in dermal fibroblasts [2]. Considering the physical properties of THG (MW: 506.5, LogP: 3.8), its penetration into the stratum corneum should be excellent (Fig. 1b). Thus, the effect of THG on keratinocytes was examined. Western blot analysis showed that treatment with THG significantly decreased the expression levels of cellular (Fig. 1C) and secreted CCN1 (Fig. 1d). It has been reported that YAP, a major downstream effector of the hippo signaling pathway, directly regulates the expression of CCN1 [16]. Therefore, we next investigated whether THG suppress CCN1 via the YAP signaling pathway. Immunocytochemical analysis revealed that the translocation of YAP to nuclei was inhibited 30 min after THG treatment, which might lead to the downregulation of CCN1 expression (Fig. 1e). These results indicate that THG is a potential suppressor of epidermal CCN1.

\section{Epidermal CCN1 regulates the balance of growth and differentiation of normal human keratinocytes. To} determine whether CCN1 is involved in the epidermal homeostasis of normal non-psoriatic skin, we first examined its localization in the skin. Immunohistochemical analysis and in situ hybridization showed that CCN1 protein and mRNA are localized at the stratum basale in the epidermis as well as in the dermis (Fig. 2a). Thus, we next investigated the function of CCN1 using knockdown technology. Secreted CCN1 was clearly decreased in siCCN1- transfected keratinocytes (Fig. 2b) and slight morphological changes 
were observed in siCCN1-transfected keratinocytes (Fig. 2c, day 4). After inducing differentiation by changing the media to a high Ca concentration and air lifting, siCCN1-transfected keratinocytes were obviously cornified compared with untreated and siCont-transfected keratinocytes (Fig. 2c, day 10). qPCR analysis showed that the expression levels of 4 differentiation-related genes, K10, Aquaporin 3 (AQP3), Transglutaminase 1 (TGM1) and Filaggrin (FLG), were significantly upregulated in siCCN1-transfected keratinocytes (Fig. 2d). Moreover, imaging cytometry revealed that the expression of Ki67, a proliferation marker, was decreased while the expression of K10 was increased in siCCN1-transfected keratinocytes (Fig. 2e). As previously reported, CCN1 is a secreted matricellular protein that is cleaved by plasmin in lung epithelial cells [17]. Thus, the ability of keratinocytes to secrete CCN1 was compared with fibroblasts. Keratinocytes treated with plasmin showed decreased levels of full length CCN1 (flCCN1: 42 $\mathrm{kDa}$ ) and secreted more cleaved CCN1 (clCCN1: $25 \mathrm{kDa}$ ) than fibroblasts (Fig. 2f). These results indicate that clCCN1 secreted from keratinocytes might affect adjacent cells in autocrine and/or paracrine dependent manners.

\section{CCN1 secreted from keratinocytes disrupts the expression of basement membrane and extracellular} matrix components. Thus, we next examined the inflammatory role of CCN1 focusing on molecules important for the basement membrane where CCN1 is highly expressed (as shown above). Keratinocytes treated with human recombinant CCN1 showed decreased expression levels of Integrin- $\beta 1$ (Fig. 3a) and increased levels of MMP9 (Fig. 3b), which may induce disruption of the basement membrane. Next, the effect of secreted CCN1 on fibroblasts was evaluated using coculture assays. qPCR analysis revealed that the expression levels of extracellular matrix components, COL1, COL3, COL5, COL7 and elastin (ELN), were significantly upregulated in fibroblasts cocultured with siCCN1-transfected keratinocytes in a knockdown efficacy dependent manner (Fig. 3c). In contrast, MMP1 expression was significantly downregulated in those fibroblasts. From these results, we hypothesized that clCCN1 secreted from keratinocytes could make the dermal extracellular matrix structure more fragile.

\section{Epidermal CCN1 is increased by UVB exposure especially in aged keratinocytes and deteriorates the} structure of elastic fibers in human skin. To further test that hypothesis, we analyzed CCN1 expression in keratinocytes and the dermal structure derived from the same human skin (Fig. 4a). Imaging cytometry revealed that aged keratinocytes had an enlarged cell size and a decreased expression level of Ki67, indicating cellular senescence $[18,19]$, compared with young keratinocytes (Fig. 4b). The expression of CCN1, which is increased in aged fibroblasts, was unchanged between young and aged keratinocytes (Fig. 4c). However, after exposure to $15 \mathrm{~mJ} / \mathrm{cm}^{2} \mathrm{UVB}$, a dose that induces oxidative stress, only aged keratinocytes showed upregulated levels of cICCN1 and evident proliferation arrest (Fig. 4b, c). To further confirm the effect of cICCN1 on the dermis, immunohistochemistry of the corresponding decolorized dermis was performed. The volume of elastic fibers was reduced in aged dermis (Fig. 4d). The expression of cICCN1, but not flCCN1 was negatively correlated with the volume of elastic fibers only in UVBirradiated keratinocytes $(p=0.076)$, which is consistent with the in vitro data (Fig. 4e). Overall, the secretion of CCN1 in the epidermis is likely to be caused by oxidative stress on aged keratinocytes, which leads to the deteriorated structures of elastic fibers. 
Treatment with THG ameliorates the structure of the basement membrane in ex vivo human skin explants. Finally, we confirmed the effects of THG on human skin explants. The treatment of skin explants with $340 \mathrm{U}$ plasmin strongly enhanced the expression level of secreted CCN1, which was significantly decreased in topically THG treated skin explants (Fig. 5a, b). Since the downregulation of CCN1 enhanced the expression of extracellular matrix components including COL7 localized at the basement membrane (as shown above), the effect of THG on collagen was evaluated. UVB exposure of skin explants induced the secretion of CCN1, which was decreased in THG-treated explants (Fig. 5c). Immunohistochemical analysis of decolorized skin revealed that the expression of $\mathrm{COL} 7$ and elastin, which is disrupted by exposure to $200 \mathrm{~mJ} / \mathrm{cm}^{2}$ UVB, was restored by the cutaneous application of THG (Fig. 5d). Taken together, the topical application of THG ameliorates the basement membrane via the downregulated secretion of CCN1 in the epidermis.

\section{Discussion}

In this study, we elucidated the function of epidermal CCN1, a factor that is important in psoriasis and in dermal senescence, and the effects of THG on the skin (shown schematically in Fig. 6). Even in normal skin, CCN1 was localized at the stratum basale and was involved in the balance of growth and differentiation. Although YAP, a transcriptional coactivator that targets CCN1 expression, is known to maintain basal epidermal stemness $[20,21]$, this study demonstrated that the regulation of CCN1 alone can switch the balance of growth and differentiation. Furthermore, in the skin of patients with psoriasis, CCN1 is also involved in the hyperplasia of keratinocytes [22]. These facts imply that the function of the YAP-CCN1 axis in normal epidermis is likely to be consistent with psoriatic epidermis.

Treatment of keratinocytes with plasmin decreased levels of intracellular flCCN1 and clCCN1 but markedly increased extracellular clCCN1 (Fig. 2f). Thus, we speculated that flCCN1 is digested intracellularly before its secretion as well as by the direct digestion of exosome-shuttled flCCN1 by extracellular plasmin [17]. Interestingly, keratinocytes secreted more clCCN1 than fibroblasts and the length of CCN1 fragments differed from each other (Fig. 2f). Because we performed western blotting to detect CCN1 using a monoclonal antibody to the N-terminus of CCN1, further studies are needed to identify the essential domain for skin senescence in the various CCN1 fragments derived from keratinocytes. Gathering these facts, epidermis-derived CCN1 is indispensable for regulating the skin microenvironment and the distribution of flCCN1 and various CCN1 fragments may control the homeostasis of skin. Next, we found that the excess secretion of CCN1 from keratinocytes affects adjacent keratinocytes and fibroblasts. Because CCN1 itself is an agonist of integrin- $\beta 1$ [23], excess CCN1 might desensitize integrin- $\beta 1$. In combination with the upregulation of MMP9, the consequent disturbance of the basement membrane might enhance the access of CCN1 to the dermis. In spite of the general upregulation of collagen in fibroblasts cocultured with siCCN1-transfected keratinocytes, we couldn't confirm the contribution of transforming growth factor- $\beta$ signaling (data not shown). Thus, it is conceivable that the disruption of extracellular matrix expression caused by epidermal clCCN1 might be mediated by the integrin-reactive oxygen species pathway [24]. 
Furthermore, we successfully demonstrated the relationship between cICCN1 and the dermal structure by analyzing isolated keratinocytes and the corresponding dermis (Fig. 3). Using that strategy, our data clearly suggest that the epidermis, which is continuously exposed to surrounding stresses, becomes vulnerable to those stresses with aging and affects tissues with a slow turnover like the dermis partly via epidermal CCN1. Future studies are needed to elucidate the behavior of CCN1 in sensitive skin and to clarify the relationship between stimuli associated with sensitive skin other than UVB and CICCN1.

For a skin care solution, we discovered that THG is a suppressor of CCN1 in keratinocytes (Figs. 4, 5). The transcription of CCN1 and the translocation of YAP are not affected by TXA (Supplemental data 1), which suggests that both TXA and THG could suppress plasmin induced CCN1 by distinct mechanisms. Since trehalose doesn't decrease CCN1 expression in keratinocytes and fibroblasts (data not shown), the two angelic acid moieties of THG must be important for the nuclear translocation of YAP. As the translocation of YAP was inhibited 30 min after THG treatment, it implies that THG might be directly involved in the upstream Hippo signaling pathway. In contrast, a previous study showed that the exogenous delivery of CCN1 or the induction of CCN1 signaling may have a therapeutic value for the treatment of fibrosis associated with wound healing [25]. Although further dermatopharmacokinetic experiments are required, THG is a promising compound for the treatment of sensitive and senescent skin.

\section{Methods}

Reagents. Antibodies used in this study were as follows: anti-CCN1 (Cat\# 14479S, Cell Signaling Technology, Danvers, MA, USA, for WB, Cat\# HPA029853, Sigma-Aldrich, St. Louis, MO, USA, for immunostaining), anti-Keratin10 (K10) (Cat\# ab76318, Abcam, Cambridge, UK), anti-Ki67 (Cat\# MU297UC, Biogenex, Fremont, CA, USA), anti-integrin 11 (Cat\# MAB1965, Sigma-Aldrich), anti-Yes-Associated Protein (YAP) (Cat\# sc-101199, Santa Cruz, Dallas, TX, USA), anti-type7 collagen (COL7) (Cat\# ab93350, Abcam), anti-elastin (Cat\# MAB2503, Abnova, Taipei, Taiwan), anti- $\beta$-actin (Cat\# sc-47778, Santa Cruz), goat anti-rabbit IgG-HRP (Cat\# A16104, Thermo Fisher Scientific, Waltham, MA, USA), goat anti-mouse IgG-HRP (Cat\# G-21040, Thermo Fisher Scientific). Alexa Fluor conjugated secondary antibodies were used for the visualization of immunostaining (Thermo Fisher Scientific). 4\% Paraformaldehyde Phosphate Buffer Solution (4\% PFA) (Cat\# 161-20141) and plasmin solution from human plasma (Cat\# 166-24231) were purchased from FUJIFILM Wako Pure Chemical Corp. (Osaka, Japan). The THG used in this study was a kind gift of NAGASE \& CO., LTD (Tokyo, Japan).

Human skin samples. Full-thickness normal human abdominal skin (from an 18 year old female) was obtained from CTI-biotech (Lyon, France) under ethical considerations. Samples are collected following informed consent of anonymized donor patients. Consenting and collecting procedures were compliant with European standards and applicable local ethical guidelines. Human eyelid skin (from 7-85 year old subjects) was provided by the Kitasato University School of Medicine (Kanagawa, Japan). The study protocol conformed to the ethical guidelines of Kitasato University's Ethics Committee (B16-285) and was conducted according to the principles of the Declaration of Helsinki. Informed consent was obtained from each patient prior to enrollment in the study. 
Cell culture. Normal human epidermal keratinocytes (NHEKs, Thermo Fisher Scientific) were maintained in GibcoTM Epilife medium, with $60 \mu \mathrm{M}$ calcium (Cat\# MEPI500CA, Thermo Fisher Scientific) supplemented with Humedia-KG supplements (Cat\# KK-6150, KURABO, Osaka, Japan). Primary human epidermal keratinocytes (HPEKp, CELLnTEC, Bern, Switzerland) were maintained in CnT-Prime Epithelial Culture Medium (Cat\# CnT-PR, CELLnTEC) or in CnT-Prime 3D Barrier Culture Medium (Cat\# CnT-PR-3D, CELLnTEC). For coculture assays, NHEKs were plated on transwell inserts and normal human dermal fibroblasts (NHDFs, Thermo Fisher Scientific) were plated on 12 well plates. Cells were maintained in EpilifeTM medium with supplements and 10\% FBS containing Dulbecco's Modified Eagle's Medium (DMEM, Cat\# 11995-065, Thermo Fisher Scientific), respectively. Eyelid skin-derived keratinocytes were obtained following treatment with Dispase $\mathbb{Z}$ and $0.25 \%$ Trypsin/EDTA. The resulting cell suspension was diluted with DMEM containing $10 \%$ FBS, then was centrifuged and plated on COL1 coated dishes for more than $24 \mathrm{~h}$. Keratinocytes obtained were then maintained as described above. Knockdown experiments were performed using Lipofectamine ${ }^{\mathrm{TM}}$ RNAiMAX Transfection Reagent (Cat\# 13778075, Thermo Fisher Scientific) and Silencer Select ${ }^{\circledR}$ Validated siRNA (Cat\#4390843: sicont, \#s7244: siCCN1-1, \#s7243: siCCN1-2, \#s7242: siCCN1-3, Thermo Fisher Scientific) according to the manufacturer's instructions. UVB irradiation was performed using a narrowband UVB lamp (Cat\# TL 20W/01 RS SLV/25, PHILIPS, Amsterdam, Netherlands) in Hanks' Balanced Salt Solution (+) after which the keratinocytes were incubated in fresh medium.

Ex vivo skin explant culture. Full-thickness normal human skin was processed within $24 \mathrm{~h}$ after excision. One cm square explants were placed on Netwell ${ }^{\mathrm{TM}}$ Inserts (Cat\# 3477, Corning, NY, USA) and put in 12 well plates. DMEM:F12 1:1 (Cat\# 11039-021, Thermo Fisher Scientific) with antibiotics added to each plate to keep the explants immersed but their surface dry (day 0 ). After the treatment with THG, plasmin or UVB, all samples were collected on day 5 . All cells and explants used in this study were incubated in a humidified atmosphere with $5 \% \mathrm{CO}_{2}$ at $37^{\circ} \mathrm{C}$.

Reverse transcriptase-qPCR. Total RNAs were isolated from cells using a RNeasy ${ }^{\circledR}$ mini kit (Cat\# 74106, QIAGEN, Hilden, Germany), followed by reverse transcription to cDNAs using a PrimeScript ${ }^{\circledR}$ RT reagent kit (Cat\# RR037A, Takara Bio, Shiga, Japan) on a SympliAmp thermal cycler (Thermo Fisher Scientific). Real-time PCR reactions were performed using Applied Biosystems ${ }^{\mathrm{TM}}$ Power SYBR ${ }^{\mathrm{TM}}$ Green PCR Master Mix (Thermo Fisher Scientific) on a QuantStudio5 ${ }^{\circledR}$ (Thermo Fisher Scientific) with the respective primers (Perfect Real Time Primer, Takara Bio). Fold-changes of expression were calculated according to the $\triangle \triangle C T$ method using ribosomal protein S18 (RPS18) as an endogenous control.

Western blotting. Whole cell extracts were prepared using $0.4 \%$ NP40 based cell lysis buffer and protein concentrations were determined using the BCA assay (Thermo Fisher Scientific). Whole cell extracts (1 $\mu \mathrm{g} /$ lane) were mixed with $4 \mathrm{x}$ sample buffer and heated at $95^{\circ} \mathrm{C}$ for $5 \mathrm{~min}$. The samples were separated by 5-20\% SDS-PAGE (Cat\# NXV-376HP, DRC, Tokyo, Japan) and transferred to polyvinylidene difluoride membranes using a Trans-blot ${ }^{\circledR}$ Turbo Transfer System (Biorad, Hercules, CA, USA). After incubation in StartingBlockTM (PBS) blocking buffer (Cat\# 37578, Thermo Fisher Scientific) at room temperature for $15 \mathrm{~min}$, the membranes were incubated with primary antibodies diluted in PBS containing $0.05 \%$ 
Tween20 (PBST) overnight at $4^{\circ} \mathrm{C}$. Proteins were visualized with corresponding secondary antibodies diluted with PBST $(1: 10,000)$ at room temperature for $1 \mathrm{~h}$, followed by chemiluminescence detection using ECL Western Blotting Detection Reagents (\#RPN2106, Cytiva, Tokyo, Japan) on LAS-4000 mini (FUJIFILM Wako Pure Chemical Corp.).

Immunocytochemistry and immunohistochemistry. Keratinocytes were fixed with 4\% PFA at room temperature for 10 min. After blocking with StartingBlockTM (PBS) blocking buffer at room temperature for 30 min, keratinocytes were incubated with anti-K10 and anti-Ki67 antibodies diluted in Can Get Signal® immunostain immunoreaction enhancer solution (Cat\# NKB-501, TOYOBO, Osaka, Japan) overnight at $4^{\circ} \mathrm{C}(1: 1,000)$. Proteins were visualized with corresponding Alexa antibodies diluted with Can Get Signal $\circledast$ immunostain immunoreaction enhancer solution (1:500), then counterstained with Alexa Fluor $^{\text {TM }} 488$ Phalloidin (Cat\# A12379, Thermo Fisher Scientific) and Hoechst 33342 at room temperature for $1 \mathrm{~h}$, followed by observation using an IN Cell Analyzer 2200 (Cytiva) and analysis on Developer toolbox (Cytiva). Skin tissues were fixed with 4\% PFA, then embedded in paraffin and cut into $5 \mu \mathrm{m}$ sections. Antigen retrieval was performed using Decloaking Chamber NxGen (BIOCARE Medical, Pacheco, $\mathrm{CA}, \mathrm{USA}$ ) at $95^{\circ} \mathrm{C}$ for $10 \mathrm{~min}$ in $\mathrm{pH} 6.0$ citrate buffer. After washing with PBS(-), slides were blocked and stained as described above using antiCCN1 and corresponding Alexa antibodies. For staining of decolorized skin, each fixed skin tissue was sectioned at a thickness of $1 \mathrm{~mm}$ in the direction of the epidermis-dermis, then washed with PBST and blocked with StartingBlock ${ }^{\text {Tm }}$ (PBS) blocking buffer. The sections were then incubated with anti-COL7 and anti-elastin antibodies diluted in StartingBlock ${ }^{\text {tw }}$ (PBS) blocking buffer. After washing, sections were visualized with Alexa antibodies and DAPI diluted in PBS(-). After further washing, these sections were subsequently decolorized by the application of Rapiclear 1.49 (Sun Jin Lab Co., Hsinchu, Taiwan). Samples were observed by FLUOVIEW FV1000 on an IX81 microscope (Olympus, Tokyo, Japan). The volume of elastic fibers was calculated using Simpleware software (JSOL Corp., Tokyo, Japan).

ELISA. The secretion of matrix metalloproteinase 9 (MMP9) into the NHEK culture medium was determined using a Human MMP-9 DuoSet ELISA (Cat\# DY911, R\&D Systems, Minneapolis, MN, USA) and was performed according to the manufacturer's instructions.

In situ hybridization. CCN1 mRNA expression levels in tissues were detected using a RNAscope ${ }^{\circledR}$ system (Advanced Cell Diagnostics, Newark, CA, USA) on $5 \mu \mathrm{m}$-sliced paraffin-embedded sections according to the manufacturer's instructions.

Nuclear translocation assay. Keratinocytes were immunostained as described above using anti-YAP and Alexa antibodies. After observation using a BZ-X810 microscope (KEYENCE, Osaka, Japan), YAP-derived intensity localized in nuclei was analyzed using a BZ-X800 analyzer (KEYENCE).

Statistical analysis. Statistical differences were assessed using EZR [26] (Saitama Medical Center, Jichi Medical University, Saitama, Japan). Values reported represent means \pm SD and $p$-values of $<0.05$ were considered statistically significant. 


\section{Declarations}

\section{Data availability}

The datasets generated during and/or analyzed during the current study are available from the corresponding author on reasonable request.

\section{Acknowledgements}

We thank NAGASE \& CO., LTD. for providing THG and Chiaki Watanabe for assisting with the experiments.

\section{Author Contributions}

M.Y. designed the study, mainly performed the experiments and wrote the manuscript. Y.K., T.N., Y.T. and S.O prepared the THG. M.Y. and Y.K. contributed equally to this work. S.I., A.T., S.K. and T.T. collected and processed skin samples. Tamie S. and Tetsuhito S. reviewed the manuscript. All authors approved the final manuscript.

\section{Additional Information}

The authors declare no competing interests.

\section{References}

[1] Nakashima, T. et al. Trehangelins A, B and C, novel photo-oxidative hemolysis inhibitors produced by an endophytic actinomycete, Polymorphospora rubra K07-0510. J. Antibiot. 66, 311-317 (2013).

[2] Kamiya, Y. et al. Study on anti-aging effects of trehangelin, a new trehalose compound. The $136^{\text {th }}$ Annual Meeting of the Pharmaceutical Society of Japan (2016).

[3] Mo, F.E. et al. CYR61 (CCN1) is essential for placental development and vascular integrity. Mol. Cell. Biol. 22, 8709-20 (2002).

[4] Lau, L.F. CCN1/CYR61: the very model of a modern matricellular protein. Cell. Mol. Life. Sci. 68, 3149$63(2011)$.

[5] Quan, T., Qin, Z., Voorhees, J.J. \& Fisher, G.J. Cysteine-rich protein 61 (CCN1) mediates replicative senescence-associated aberrant collagen homeostasis in human skin fibroblasts. J. Cell. Biochem. 113, 3011-8 (2012).

[6] Qin, Z., Fisher, G.J. \& Quan, T. Cysteine-rich protein 61 (CCN1) domain-specific stimulation of matrix metalloproteinase-1 expression through aVß3 integrin in human skin fibroblasts. J. Biol. Chem. 288, 12386-12394 (2013). 
[7] Qin, Z., Okubo, T., Voorhees, J.J., Fisher, G.J. \& Quan, T. Elevated cysteine-rich protein 61 (CCN1) promotes skin aging via upregulation of IL-1 $\beta$ in chronically sun-exposed human skin. Age (Dordr). 36, 353-364 (2014).

[8] Quan, T. \& Fisher G.J. Role of Age-Associated Alterations of the Dermal Extracellular Matrix Microenvironment in Human Skin Aging: A Mini-Review. Gerontology. 61, 427-434 (2015).

[9] Sun, Y. et al. CCN1 promotes IL-1 $\beta$ production in keratinocytes by activating p38 MAPK signaling in psoriasis. Sci. Rep. 7, 43310 (2017).

[10] Wu, P. et al. Cyr61/CCN1 is involved in the pathogenesis of psoriasis vulgaris via promoting IL-8 production by keratinocytes in a JNK/NF-KB pathway. Clin. Immunol. 174, 53-62 (2017).

[11] Li, H. et al. Cyr61/CCN1 induces CCL20 production by keratinocyte via activating p38 and JNK/AP-1 pathway in psoriasis. J. Dermatol. Sci. 88, 46-56 (2017).

[12] Honari, G. Andersen, R. \& Maibach, H. Sensitive Skin Syndrome, 2nd edition (CRC Press, 2017).

[13] Brenaut, E., Misery, L. \& Taieb, C. Sensitive Skin in the Indian Population: An Epidemiological Approach. Front. Med. 6, 29 (2019).

[14] Farage, M.A. The Prevalence of Sensitive Skin. Front. Med. 6, 98 (2019).

[15] Misery, L., Loser, K. \& Ständer, S. Sensitive skin. J. Eur. Acad. Dermatol. Venereol. 30, Suppl 1, 2-8 (2016).

[16] Quan, T., Johnston, A., Gudjonsson, J.E. \& Fisher, G.J. CYR61/CCN1: A Novel Mediator of Epidermal Hyperplasia and Inflammation in Psoriasis? J. Invest. Dermatol. 135, 2562-2564 (2015).

[17] Moon, H.G. et al. CCN1 secretion and cleavage regulate the lung epithelial cell functions after cigarette smoke. Am. J. Physiol. Lung. Cell. Mol. Physiol. 307, 326-337 (2014).

[18] Liao, Y.H. et al. Determination of chronological aging parameters in epidermal keratinocytes by in vivo harmonic generation microscopy. Biomed. Opt. Express. 4, 77-88 (2013).

[19] Gorgoulis, V. et al. Cellular Senescence: Defining a Path Forward. Cell, 179, 813-827 (2019).

[20] Zhang, H., Pasolli, H.A. \& Fuchs, E. Yes-associated protein (YAP) transcriptional coactivator functions in balancing growth and differentiation in skin. Proc. Natl. Acad. Sci. U S A. 108, 2270-2275 (2011).

[21] Walko, G. et al. A genome-wide screen identifies YAP/WBP2 interplay conferring growth advantage on human epidermal stem cells. Nat. Commun. 8, 14744 (2017).

[22] Sun, Y. et al. CCN1, a Pro-Inflammatory Factor, Aggravates Psoriasis Skin Lesions by Promoting Keratinocyte Activation. J. Invest. Dermatol. 135, 2666-2675 (2015). 
[23] Leu, S.J. et al. Identification of a novel integrin alpha 6 beta 1 binding site in the angiogenic inducer CCN1 (CYR61). J. Biol. Chem. 278, 33801-33808 (2003).

[24] Chen, C.C. et al. Cytotoxicity of TNFalpha is regulated by integrin-mediated matrix signaling. EMBO J. 26, 1257-1267 (2007).

[25] Jun, J.I. \& Lau, L.F. The matricellular protein CCN1 induces fibroblast senescence and restricts fibrosis in cutaneous wound healing. Nat. Cell. Biol. 12, 676-685 (2010).

[26] Kanda, Y. Investigation of the freely available easy-to-use software 'EZR' for medical statistics. Bone Marrow Transplant. 48, 452-458 (2013).

Figures 


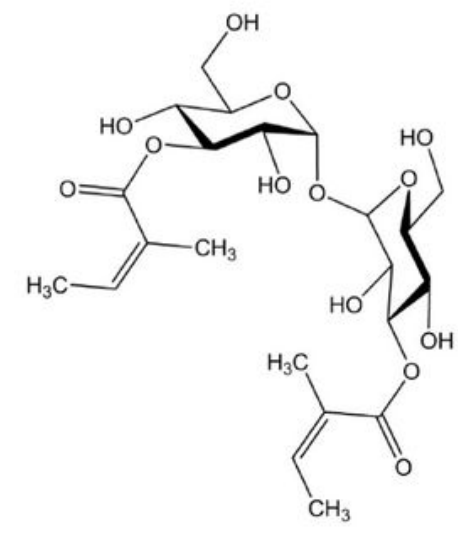

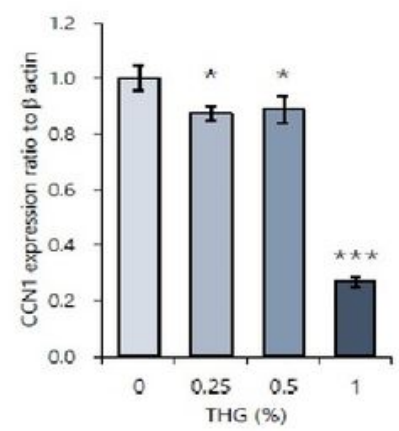

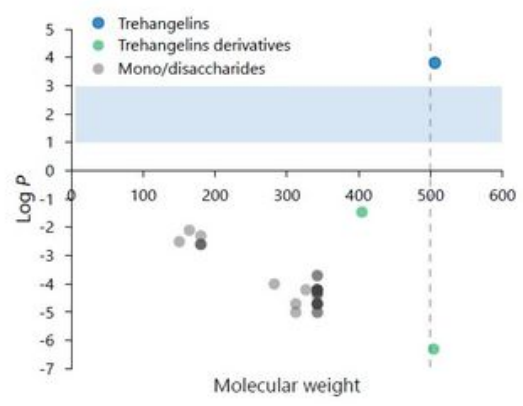
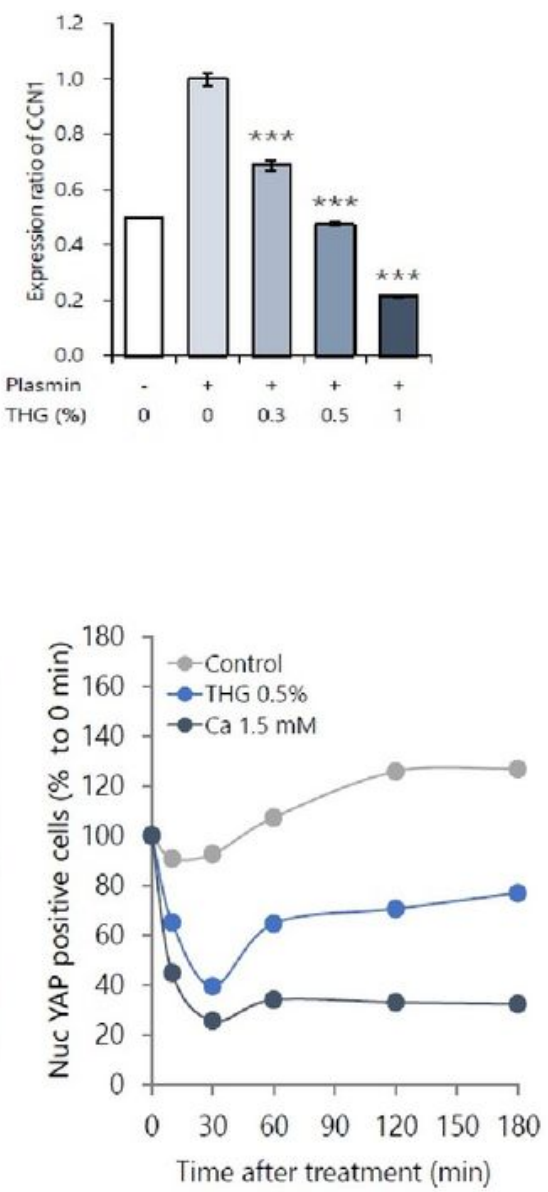

\section{Figure 1}

Trehangelins (THG) suppress the expression of CCN1 in keratinocytes by inhibiting the translocation of Yes-associated protein (YAP) to the nucleus. Structure of THG (a). Estimated LogP of THG, THG derivatives and mono/disaccharides (b). Effects of THG on CCN1 expression. Keratinocytes were pretreated with THG for $24 \mathrm{~h}$ and then were stimulated with or without $10 \mathrm{U}$ plasmin, after which they were incubated for another $24 \mathrm{~h}$. The expression of cellular CCN1 (c) and secreted CCN1 (d) was 
determined by Western Blotting and was quantitate. Values reported are means $\pm S D$ of $n=3$ replicates, Dunnett's test, ${ }^{*} p<0.05,{ }^{*} p<0.01,{ }^{*} * * p<0.001$ (c\&d). Immunocytochemistry of YAP and analysis of nuclear YAP-positive cells $0-3 \mathrm{~h}$ after $\mathrm{Ca}$ or THG treatment (e). Values reported are means of $n=10,000$ cells, Bar $=$ $100 \mu \mathrm{m}(\mathrm{e})$.
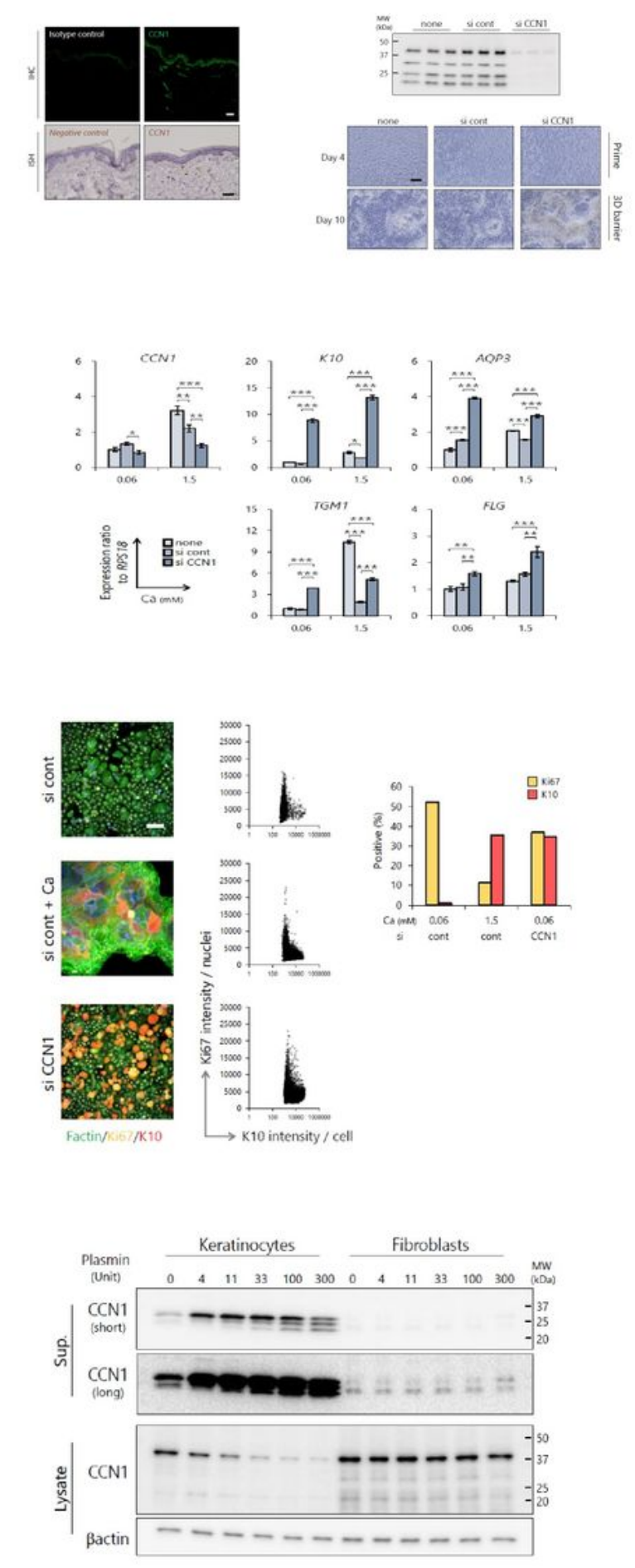

Figure 2 
Epidermal CCN1 regulates the balance of growth and differentiation of normal human keratinocytes. CCN1 localization examined by immunohistochemistry (IHC) and by in situ hybridization (ISH) in human skin. Bar $=100 \mu \mathrm{m}$ (a). Knockdown efficacy of CCN1 on day 4 was determined by WB of HPEKp supernatant samples. Full-length gels and blots are included in a Supplementary Information file. (b). Apical view of reconstructed skin before (day 4) and after (day 10) induction of differentiation, Bar $=300$ $\mu \mathrm{m}$ (c). Effects of CCN1 knockdown on mRNA levels of CCN1, Keratin 10, Aquaporin 3, Transglutaminase 1 and Filaggrin determined by qPCR. Total RNAs were collected from siRNA transfected keratinocytes $6 \mathrm{~h}$ after Ca stimulation. Values reported are means $\pm S D$ of $n=3$ replicates, Tukey's test, ${ }^{*} p<0.05,{ }^{* \star} p<0.01$, ${ }^{* * *} \mathrm{p}<0.001$ (d). Effects of CCN1 knockdown on Ki67 and K10 expression determined by imaging cytometry. Keratinocytes were fixed $48 \mathrm{~h}$ after transfection, Bar $=100 \mu \mathrm{m}, \mathrm{n}=10,000$ cells (e). Comparison of reactivity to plasmin treatment between keratinocytes and fibroblasts. Secreted and cellular CCN1 levels were determined by WB. Samples were collected $24 \mathrm{~h}$ after plasmin treatment. Full-length gels and blots are included in a Supplementary Information file (f). 

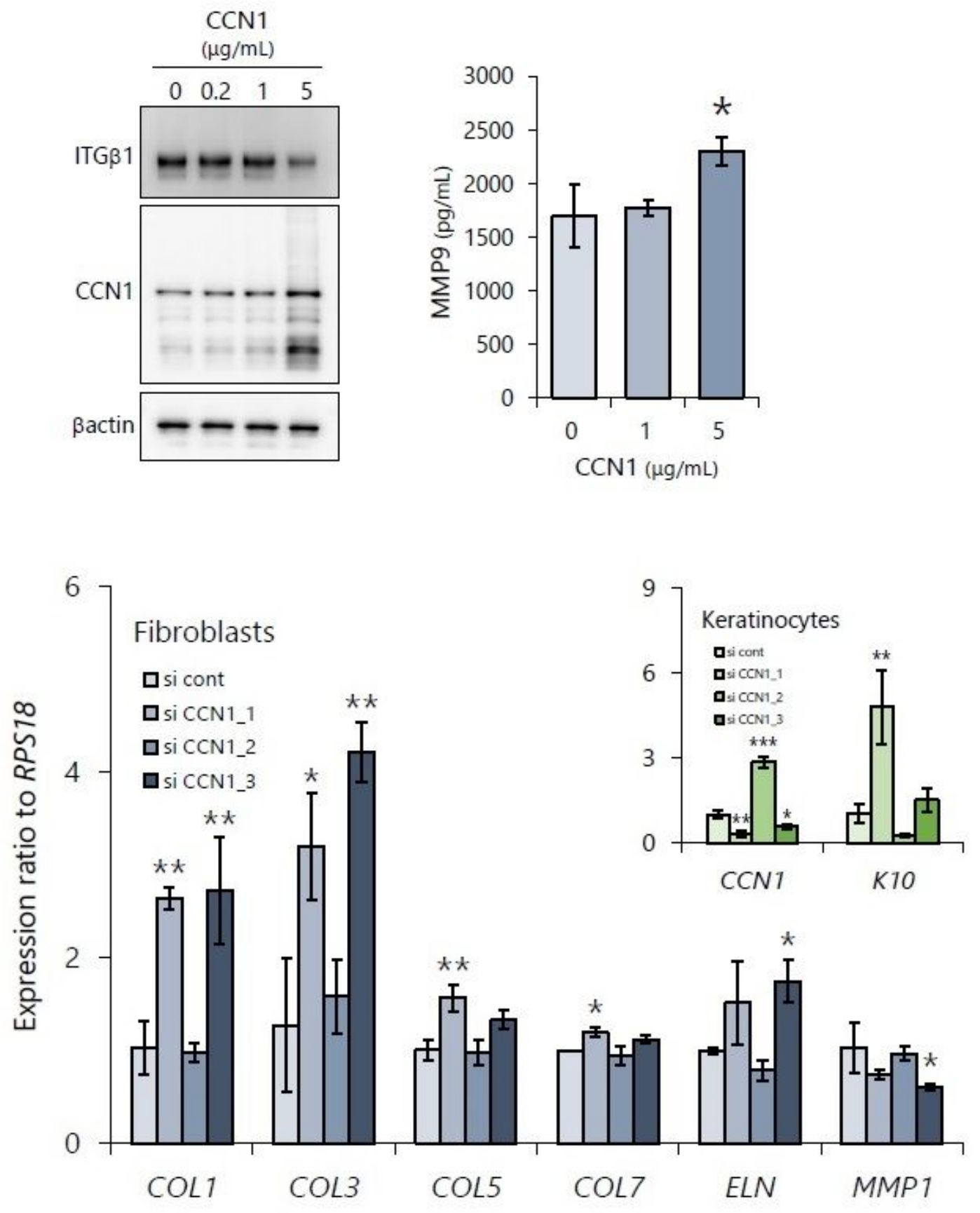

Figure 3

Secreted CCN1 from keratinocytes disrupts the expression of basement membrane and extracellular matrix components. Effects of excess CCN1 on inflammation of the stratum basale. Integrin- $₫ 1$ and MMP9 expression in keratinocytes treated with human recombinant CCN1 for $48 \mathrm{~h}$ determined by western blotting and ELISA, respectively. Full-length gels and blots are included in a Supplementary Information file ( $a$ and b). Effects of keratinocyte-derived CCN1 on mRNA expression levels of COL1, COL3, COL5, 
COL7, ELN and MMP1, in fibroblasts determined by qPCR (c). Values reported are means \pm SD of $n=3$ replicates, Dunnett's test, ${ }^{*} p<0.05,{ }^{* *} p<0.01,{ }^{* \star *} p<0.001$.
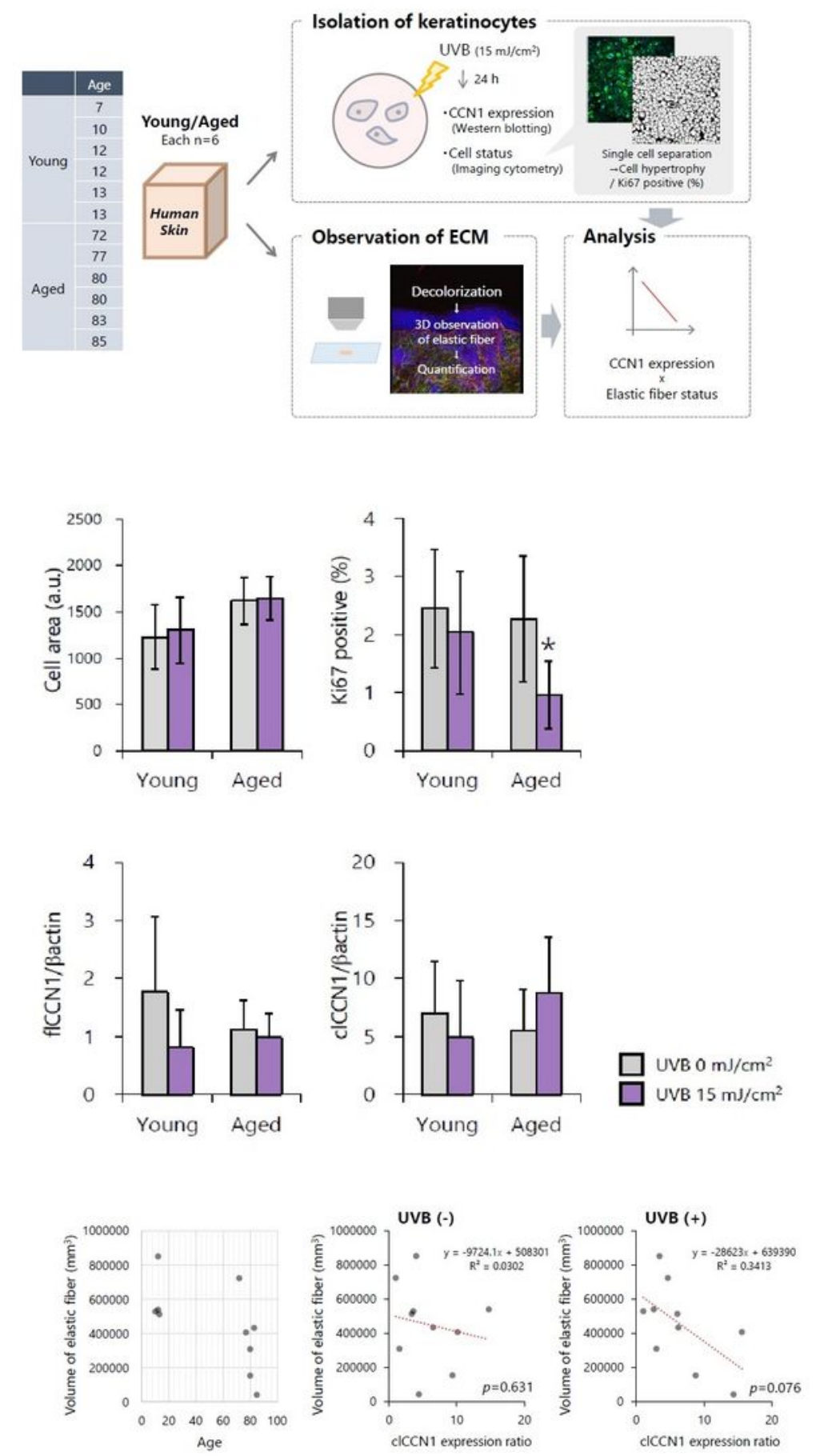

Figure 4

Epidermal CCN1 is increased by UVB exposure especially in aged keratinocytes and deteriorates the structure of elastic fibers in human skin. Overview of the experimental method (a). Cell area and Ki67positive rate of isolated keratinocytes determined by imaging cytometry (b) and CCN1 expression in 
keratinocytes determined by WB $24 \mathrm{~h}$ after 0 or $15 \mathrm{~mJ} / \mathrm{cm} 2 \mathrm{UVB}$ irradiation. Values reported are means \pm $S D$ of $n=6$, Student's $t$ test (c). Volume of elastic fibers determined by immunohistochemistry of decolorized skin and structural analysis (d). Correlation analysis between clCCN1 expression and the volume of elastic fibers performed by Pearson's correlation test, $n=10(e)$.

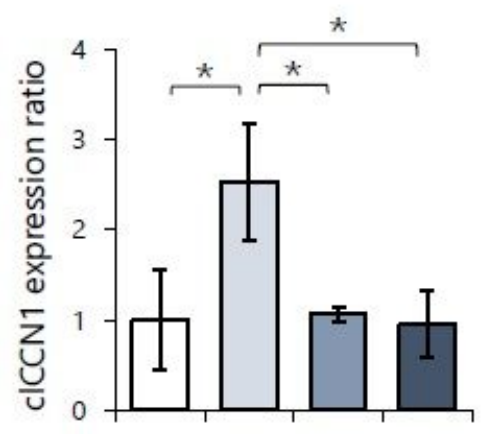

Plasmin THG (\%) $\quad 0 \quad 00.1 \quad 1$

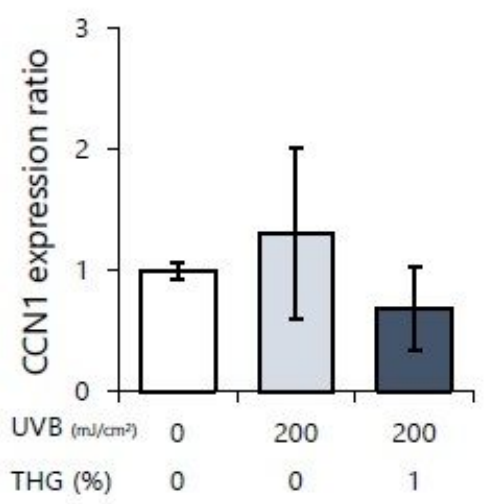

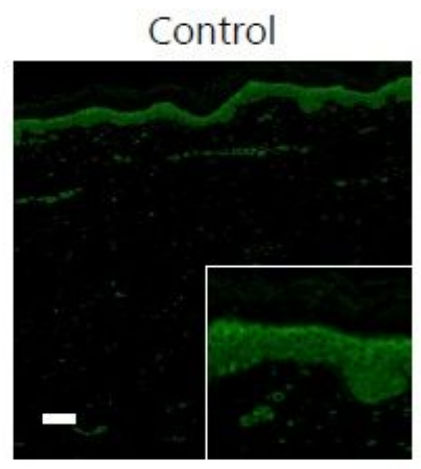
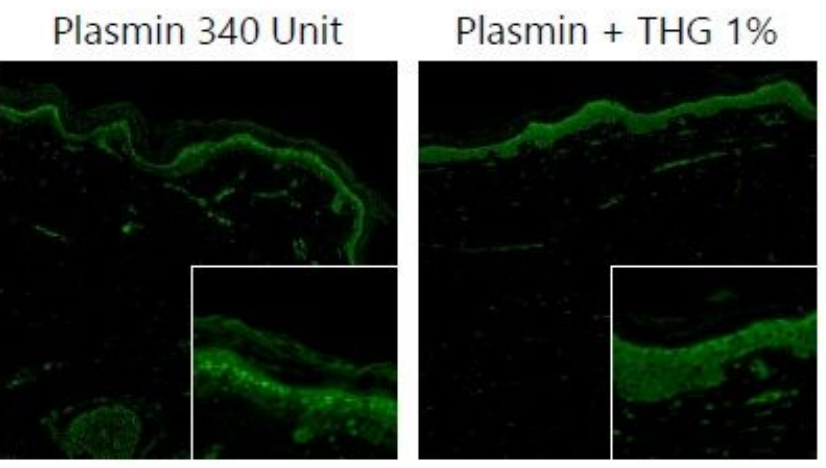

CCN1

UVB $200 \mathrm{~mJ} / \mathrm{cm}^{2}$

Control

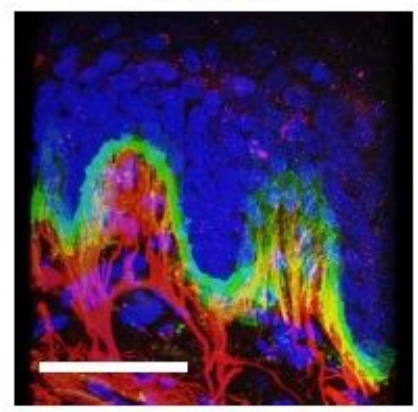

UVB $200 \mathrm{~mJ} / \mathrm{cm}^{2}$
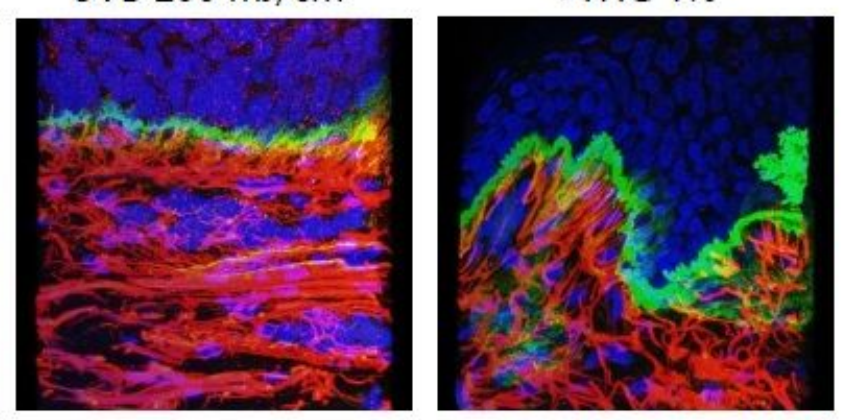

DAPI/COL7/ELN

\section{Figure 5}

Treatment with THG ameliorates the structure of the basement membrane in ex vivo human skin explants. Effects of topically applied THG on CCN1 expression in plasmin-treated ex vivo skin explants. On day 1, 0-1\% THG was applied on the stratum corneum and $340 \mathrm{U}$ plasmin was systematically applied to the medium. cICCN1 in the supernatant was determined by WB. Values reported are means \pm SD of $n=3$ replicates, Tukey's test, * $\mathrm{p}<0.05$ (a). CCN1 expression and localization was determined by immunohistochemistry, Bar $=100 \mu \mathrm{m}$ (b). Effects of topically applied THG on COL7 and ELN structure in UVB irradiated ex vivo skin explants. $1 \%$ THG was topically treated on day 1 . On days 1,2 and 3 , the inserts were transferred to 12 well plates with PBS(-) and were exposed to $200 \mathrm{~mJ} / \mathrm{cm} 2 \mathrm{UVB}$, after which they were put back into their original wells. Cellular CCN1 was determined by WB. Values reported are means \pm SD of $n=3$ replicates, Tukey's test (c). COL7 and ELN expression and localization were determined by immunohistochemistry of decolorized skin explants, Bar $=50 \mu \mathrm{m}$ (d). 


\section{Features of sensitive skin}

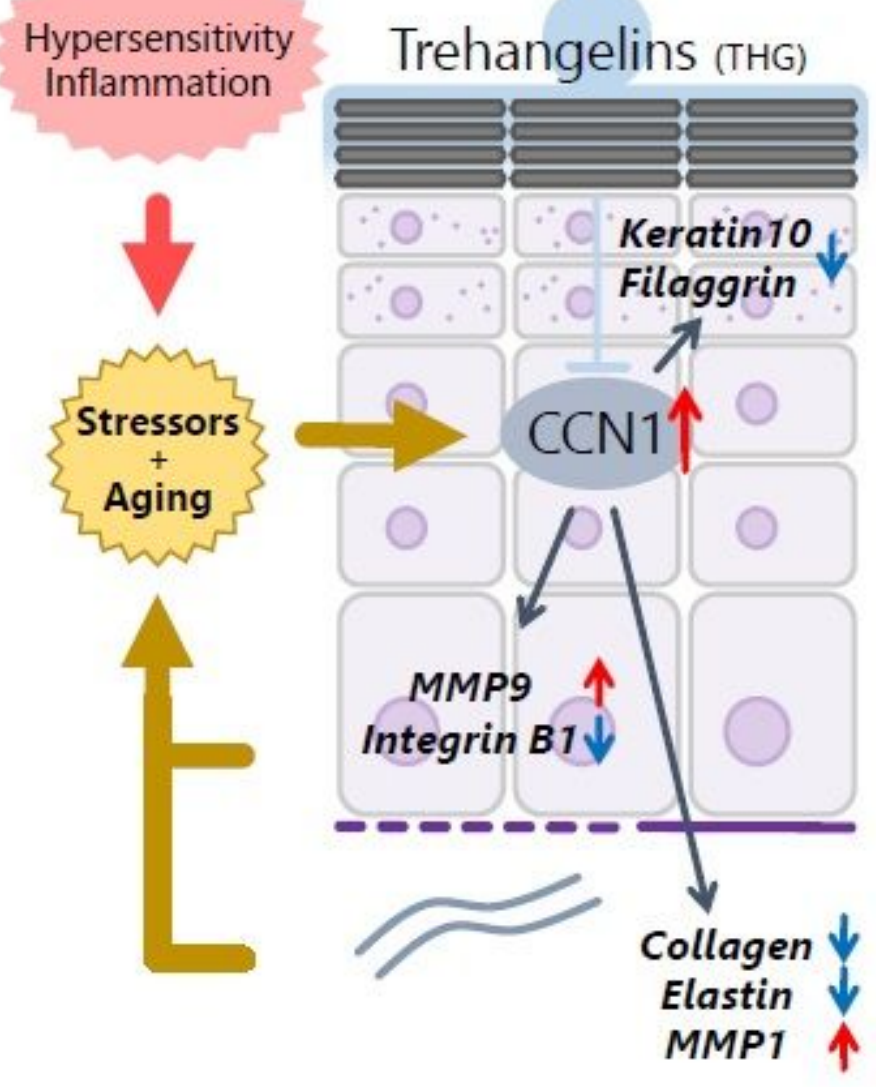

Figure 6

Graphical model of CCN1 driven aged and sensitive skin and the effect of THG.

\section{Supplementary Files}

This is a list of supplementary files associated with this preprint. Click to download.

- 20210728THGCCN1yokotaS1.pdf 